\title{
Recent heavy flavor results from STAR
}

\author{
André Mischke* for the STAR Collaboration ${ }^{\dagger}$ \\ Institute for Subatomic Physics, Utrecht University, \\ Princetonplein 5, 3584 CC Utrecht, \\ The Netherlands. \\ E-mail: a.mischke@phys.uu.nl
}

\begin{abstract}
We report on recent heavy flavor measurements from the STAR experiment at RHIC 1 . The measured charm cross section in heavy-ion collisions scales with the number of binary collisions, which is an indication for exclusive charm production in the initial state of the collision. The observed strong suppression of non-photonic electrons at high $p_{\mathrm{T}}$ in $\mathrm{Au}+\mathrm{Au}$ collisions together with the azimuthal correlation measurements in $\mathrm{p}+\mathrm{p}$ collisions imply a suppression of $B$ production in heavy-ion collisions. We also present recent measurements of the $\Upsilon$ cross section in $\mathrm{p}+\mathrm{p}$ collisions.
\end{abstract}

\section{Introduction}

The fundamental theory of strong interactions, Quantum Chromodynamics (QCD), predicts a phase transition from hadronic matter to a system of deconfined quarks and gluons, the Quark Gluon Plasma, if the surrounding temperature exceeds a critical value. The goal of heavy-ion physics is to produce such a deconfined QCD state and to study its properties under controlled laboratory conditions. The accelerator with the current highest collision energy for atomic nuclei is the Relativistic Heavy-Ion Collider (RHIC) at Brookhaven National Laboratory. Current results from the RHIC experiments have given compelling evidences that the produced medium is indeed a plasma of quarks and gluons, but it behaves like a "perfect" fluid rather than an ideal gas 2, 3. One of the intriguing results is the strong modification of the jet structure inside the created medium. Theoretical model calculations that attribute the jet attenuation to the energy loss of partons traversing through the medium have successfully described the present data.

The study of heavy flavor (charm, bottom) production in heavy-ion collisions provides key tests of the parton energy loss mechanisms and offers important information on the properties of the produced medium. Due to their large mass, heavy quarks are expected to be primarily produced in the early stage of the collision and, therefore, probe the complete space-time evolution of the medium. Theoretical models predicted that heavy quarks should experience smaller energy loss than light quarks while propagating through the QCD medium due to the suppression of small angle gluon radiation (dead-cone effect) [4].

\section{Recent results}

The charm cross section at mid-rapidity is determined from measurements of open charm mesons and from the reconstruction of heavy flavor semi-leptonic decays via muon and electron measurements. These three measurements, which are performed by the STAR Time

\footnotetext{
${ }^{*}$ The author is grateful for the support by the Netherlands Organisation for Scientific Research (NWO).

${ }^{\dagger}$ For the full author list, see [8].
} 

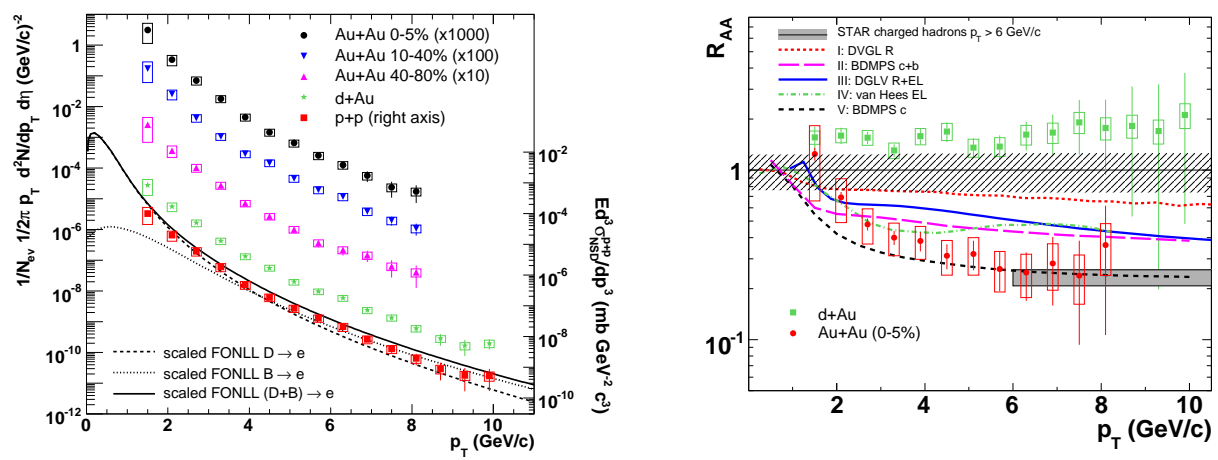

Figure 1: Left panel: Non-photonic electron spectra in $\mathrm{p}+\mathrm{p}, \mathrm{d}+\mathrm{Au}$ and, for different centralities, in $\mathrm{Au}+\mathrm{Au}$ collisions at $\sqrt{s_{\mathrm{NN}}}=200 \mathrm{GeV}$. The curves are pQCD predictions scaled by 5.5 for $\mathrm{p}+\mathrm{p}$ collisions. The right axis gives the cross section for the $\mathrm{p}+\mathrm{p}$ spectrum. Right panel: Nuclear modification factor $R_{A A}$ for $\mathrm{d}+\mathrm{Au}$ and $\mathrm{Au}+\mathrm{Au}$ collisions. The curves correspond to different model predictions as indicated in the figure. The error bars (boxes) indicates the statistical (uncorrelated systematic) errors. The dashed box illustrates the overall normalization uncertainty.

Projection Chamber (TPC) and Time-of-Flight (ToF) detector, cover 95\% of the cross section. $D^{0}$ mesons are reconstructed in the hadronic decay channel $D^{0} \rightarrow K^{-} \pi^{+}$by calculating the invariant mass of all oppositely charged TPC track combinations [5, 6. The decay particles are identified using the specific energy loss $(d E / d x)$ measured in the TPC. Muons at low $p_{\mathrm{T}}(<250 \mathrm{MeV} / \mathrm{c})$ are identified by the combination of the $m^{2}$ measurement in the ToF detector and the specific energy loss $(d E / d x)$ measurement in the TPC. A cut on the distance of closest approach is used to separate the prompt from decay muons. The non-photonic electrons are obtained by combining the $d E / d x$ and ToF $(|1 / \beta-1|<0.03)$ measurement. A description of the determination of the photonic electron background, the applied corrections and the procedure to calculate the charm cross section from the $p_{\mathrm{T}}$ spectra of $D^{0}, \mu$ and $e$ is given in [5]. The obtained cross section is found to be $\sigma_{c c}^{N N}=1.40 \pm 0.11 \pm 0.39 \mathrm{mb}$ in the $12 \%$ most central $\mathrm{Au}+\mathrm{Au}$ collisions. NLO calculations predict a factor of $\approx 5$ smaller value for the cross section. More precise measurements are required in order to understand this discrepancy. Moreover, the cross section at mid-rapidity shows binary collision scaling which is an indication for charm production exclusive in the initial state of the collisions 7 . Hence, there is no room for thermal charm production in the medium.

Electrons at higher $p_{\mathrm{T}}(>4 \mathrm{GeV} / \mathrm{c})$ are identified by a combined measurement using the TPC and the Electromagnetic Calorimeter (EMC). The analysis details are described in 8]. Fig. 1 (left) shows the $p_{\mathrm{T}}$ spectrum of non-photonic electrons in $\mathrm{p}+\mathrm{p}, \mathrm{d}+\mathrm{Au}$ and, for different centralities, in $\mathrm{Au}+\mathrm{Au}$ collisions at $\sqrt{s_{\mathrm{NN}}}=200 \mathrm{GeV}$, which are measured up to $10 \mathrm{GeV} / \mathrm{c}$. A pQCD calculation for heavy quarks production in $\mathrm{p}+\mathrm{p}$ collisions [9], indicated in Fig. 1 (left) by the solid line, describes the overall shape of the $p_{\mathrm{T}}$ distribution but it has the same scaling discrepancy as observed for the charm cross section measurement. Nuclear effects are usually quantified in the nuclear modification factor $R_{A A}$ where the yield in $\mathrm{Au}+\mathrm{Au}$ is divided by the yield in $\mathrm{p}+\mathrm{p}$ scaled by the number of binary collisions. The non-photonic electron yield exhibits an unexpectedly large suppression in central $\mathrm{Au}+\mathrm{Au}$ collisions at high $p_{\mathrm{T}}$, suggesting substantial energy loss of heavy quarks in the produced medium (cf. Fig. 1 right). The suppression factor has a similar value as observed for light 

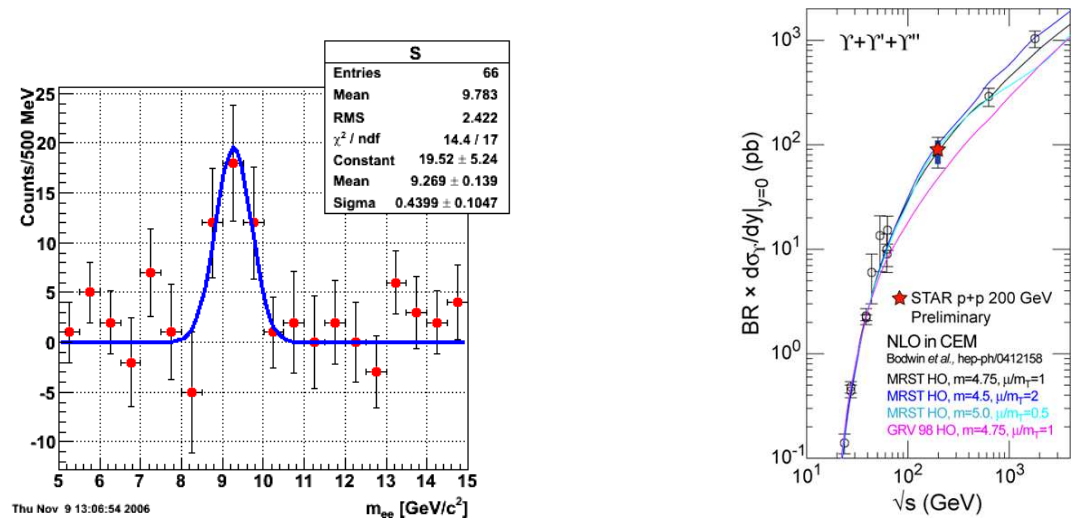

Figure 2: Left panel: Background subtracted di-electron invariant mass distribution in $\mathrm{p}+\mathrm{p}$ collisions at $\sqrt{s_{\mathrm{NN}}}=200 \mathrm{GeV}$. The blue line indicates a Gaussian fit to the data. Right panel: Excitation function of the $\Upsilon$ cross section. The data is compared to previous measurements and NLO calculations. The curves of the NLO calculations are scaled by a factor of 1.44 to account for the excited states.

quark hadrons in central $\mathrm{Au}+\mathrm{Au}$ collisions, indicated by the grey box in the figure. The data is compared to different energy loss models [10, 11, 12, 13, which vary essentially in the interaction processes and energy loss mechanisms taken into account. As indicated in Fig. 1 (right), all models underpredict the measured suppression factor at high $p_{\mathrm{T}}$. It has been shown that the data is described reasonably well if the models assume electrons from $D$ decays only. Therefore, the observed discrepancy could indicate that the $B$ dominance over $D$ mesons starts at higher $p_{\mathrm{T}}$. A possible scenario for $B$ meson suppression invokes collisional dissociation in the medium [14].

To verify the $B$ production dominance at higher $p_{\mathrm{T}}$ one has to disentangle the $D$ and $B$ contribution to the non-photonic electron spectrum experimentally. Recent results on measurements in $\mathrm{p}+\mathrm{p}$ collisions of the azimuthal angular correlations between electrons (from heavy-flavor decays) associated with charged hadrons have shown that the relative $B$ contribution, $B /(B+D)$, is about $40 \%$ at $p_{\mathrm{T}}=5 \mathrm{GeV} / \mathrm{c}\left[15\right.$. The measured $p_{\mathrm{T}}$ dependence of the relative $B$ contribution can be used to verify the input parameters for most of the energy loss models. First results on a different approach show the proof of principle to disentangle the $D$ and $B$ contributions to the non-photonic electrons using electron- $D^{0}$ meson azimuthal correlations [16].

The suppression of heavy quarkonium states provides an essential tool to study the temperature of the medium. The large acceptance $(|\eta|<1$ and $0<\phi<2 \pi)$ of the STAR TPC and EMC allows the measurement of $\Upsilon$ production at mid-rapidity. The $\Upsilon$ is reconstructed through the $\Upsilon \rightarrow e^{+} e^{-}$decay channel. Both detectors have very good electron identification capabilities and allow the combined measurement of the momentum (TPC) and the energy (EMC) of the decay electrons. Details of the data analysis can be found in [17. Due to the finite momentum resolution of the TPC, individual $\Upsilon$ states, 1S, $2 \mathrm{~S}$ and $3 \mathrm{~S}$, can not be resolved. The EMC serves as a trigger for high momentum electrons utilizing two dedicated trigger settings 18 . The presented data are from the 2006 Run with an integrated luminosity of $\mathcal{L}=5.6 \mathrm{pb}^{-1}$. The invariant mass distribution of unlike-sign electron pairs is shown in Fig. 2 (left). The peak width is consistent with the expected mass 
resolution. The corrections applied to the raw yield are discussed in [17. The obtained production cross-section of $B R_{e e} \times \frac{d \sigma}{d y}{ }_{y=0}=91 \pm 28$ (stat.) \pm 22 (sys.) pb follows the world data trend (cf. Fig. 2 right). Within uncertainties, the data shows very good agreement with NLO calculations. The low cross section of the $\Upsilon$ at RHIC energies make this a luminosity limited measurement. The upcoming measurement in heavy-ion collisions will shed more light into the expected melting of quarkonia states in the hot and dense medium and provide an estimate of the medium temperature.

\section{Summary and conclusions}

In this paper, we summarize recent heavy flavor results from the STAR experiment at RHIC. The charm cross-section was extracted from a combined fit to the measured spectra of open charm mesons, electrons and muons both from semi-leptonic heavy flavor decays. The charm cross section in $\mathrm{Au}+\mathrm{Au}$ collisions scales with the number of binary collisions supporting the assumption that charm is exclusively produced in the initial state of the collision and that there is no room for thermal production in the medium. The suppression of the nuclear modification factor of non-photonic electrons at high $p_{\mathrm{T}}$ in $\mathrm{Au}+\mathrm{Au}$ collisions is much larger than expected. The theoretical explanations are yet inconclusive. First results on azimuthal angular correlations of non-photonic electrons and hadrons ( $D^{0}$ mesons) in $\mathrm{p}+\mathrm{p}$ collisions show its ability to disentangle the $D$ and $B$ contribution to the electron spectrum. The recent completion of the STAR EMC allowed the first measurement of $\Upsilon$ production at midrapidity in $\mathrm{p}+\mathrm{p}$ collisions. The $\Upsilon$ cross section is consistent with pQCD calculations and the world data trend. More exciting results are about to come with the STAR detector upgrades

(full barrel Time-of-Flight and Heavy Flavor Tracker) which will allow direct measurements of the nuclear modification factor of $D$ and $B$ mesons in heavy-ion collisions.

\section{References}

[1] Slides:

http://indico. cern. ch/contributionDisplay.py? contribId=199\&amp; sessionId=5\&amp; conf Id=9499

[2] B.I. Abelev et al. (STAR Collaboration), Nucl. Phys. A757, 102 (2005).

[3] P. Jacobs and X.N. Wang, Prog. Part. and Nucl. Phys. 54, 443 (2005).

[4] Y.L. Dokshitzer and D.E. Kharzeev, Phys. Lett. B519, 199 (2001).

[5] J. Adams et al. (STAR Collaboration), Phys. Rev. Lett. 94, 062301 (2005).

[6] H. Zhang et al. (STAR Collaboration), Nucl. Phys. A774, 701 (2006).

[7] Y. Zhang et al. (STAR Collaboration), J. Phys. G32, S529 (2006).

[8] B.I. Abelev et al. (STAR Collaboration), Phys. Rev. Lett. 98, 192301 (2007).

[9] M. Cacciari, P. Nason and R. Vogt, Phys. Rev. Lett. 95, 122001 (2005).

[10] M. Djordjevic et al., Phys. Lett. B632, 81 (2006).

[11] N. Armesto et al., Phys. Lett. B637, 362 (2006).

[12] S. Wicks et al., Nucl. Phys. A784, 426, (2007).

[13] H. van Hees, V. Greco and R. Rapp, Phys. Rev. C73, 034913 (2006).

[14] A. Adil and I. Vitev, Phys. Lett. B649, 139 (2007).

[15] X. Lin et al. (STAR Collaboration), submitted to J. Phys. G (arXiv: nucl-ex/0701050).

[16] A. Mischke et al. (STAR Collaboration), arXiv: 0705.2089.

[17] M.R. Cosentino et al. (STAR Collaboration), arXiv: 0706.0892.

[18] P. Djawotho et al. (STAR Collaboration), submitted to J. Phys. G (arXiv: nucl-ex/0701075). 